\title{
Analytic Result for a Two-Loop Five-Particle Amplitude
}

\author{
D. Chicherin, ${ }^{1}$ T. Gehrmann, ${ }^{2}$ J. M. Henn, ${ }^{1}$ P. Wasser, ${ }^{3}$ Y. Zhang, ${ }^{1}$ and S. Zoia ${ }^{1}$ \\ ${ }^{1}$ Max-Planck-Institut für Physik, Werner-Heisenberg-Institut, D-80805 München, Germany \\ ${ }^{2}$ Physik-Institut, Universität Zürich, Wintherturerstrasse 190, CH-8057 Zürich, Switzerland \\ ${ }^{3}$ PRISMA Cluster of Excellence, Johannes Gutenberg University, D-55099 Mainz, Germany
}

(Received 28 December 2018; published 29 March 2019)

\begin{abstract}
We compute the symbol of the full-color two-loop five-particle amplitude in $\mathcal{N}=4$ super Yang-Mills theory, including all nonplanar subleading-color terms. The amplitude is written in terms of permutations of Parke-Taylor tree-level amplitudes and pure functions to all orders in the dimensional regularization parameter, in agreement with previous conjectures. The answer has the correct collinear limits and infrared factorization properties, allowing us to define a finite remainder function. We study the multi-Regge limit of the nonplanar terms, analyze its subleading power corrections, and analytically present the leading logarithmic terms.
\end{abstract}

DOI: 10.1103/PhysRevLett.122.121602

Introduction.-The study of scattering amplitudes in maximally supersymmetric Yang-Mills theory $(\mathcal{N}=4 \mathrm{SYM})$ has brought about many advances in quantum field theory (QFT). Experience shows that having analytical "data," i.e., explicit results, for amplitudes available is vital to find structures and patterns in seemingly complicated results, and to test new ideas. Cases in point are dual-conformal symmetry [1-3], the symbol analysis [4], insights of Regge limits in perturbative QFT [5], and the structure of infrared divergences [6,7], just to name a few.

Thanks to recent progress, an abundant wealth of data is available for planar scattering amplitudes in $\mathcal{N}=4 \mathrm{SYM}$ theory. Up to five particles, the functional form of the latter is fixed by dual conformal symmetry $[8,9]$, in agreement with previous conjectures $[7,10]$. Starting from six particles, there is the freedom of a dual conformally invariant function $[1,11,12]$, which has been the subject of intense study.

Conjecturally, the function space of the latter is known in terms of iterated integrals, or symbols. Using bootstrap ideas, perturbative results at six and seven particles have been obtained at high loop order [13-18]. This led, in particular, to insight into how the Steinmann relations are realized in perturbative QFT [19], and to intriguing observations about a possible cluster algebra structure of the amplitudes [20].

On the other hand, few results are available to date beyond the planar limit. The four-particle amplitude is

Published by the American Physical Society under the terms of the Creative Commons Attribution 4.0 International license. Further distribution of this work must maintain attribution to the author(s) and the published article's title, journal citation, and DOI. Funded by SCOAP ${ }^{3}$. known to three loops [21], and no results are available beyond one loop for more than four particles. In order to study whether properties such as integrability, hidden dual conformal symmetry, and properties of the function space generalize to the full theory, it is crucial to have more data. In this Letter, we compute, in terms of symbols, a full fiveparticle scattering amplitude in QFT. While all the required planar master integrals are already known analytically in the literature, one nonplanar integral family was still missing, up to now. We fill this gap and discuss its calculation in a dedicated parallel paper [22].

Calculation of the master integrals.-The integral topologies needed for massless five-particle scattering at two loops are shown in Fig. 1. The integrals in four-point kinematics, Figs. 1(d)-1(f), are known from Refs. [23,24]. The master integrals of the planar topology depicted in Fig. 1(a) were computed in Refs. [25-27], whereas the nonplanar integral family shown in Fig. 1(b) was computed in Ref. [28] (see also [29-32]). We devote a parallel paper [22] to the calculation of the missing nonplanar family,
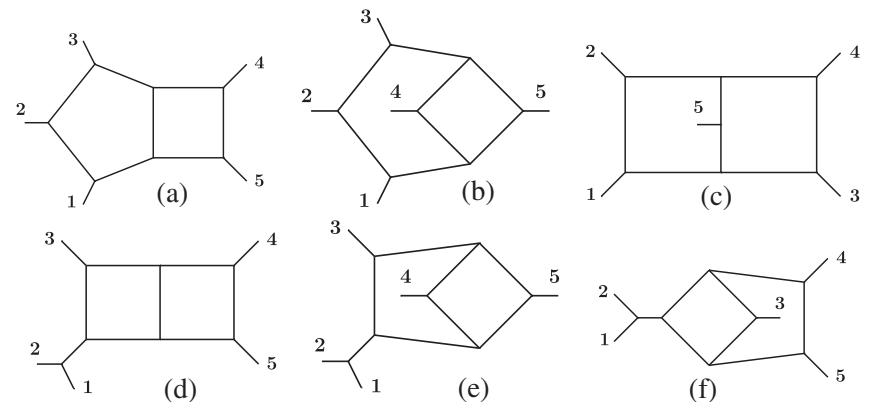

FIG. 1. Diagrams in the representation of [33] of the integrand of the two-loop five-point amplitude in $\mathcal{N}=4$ SYM. We omit the associated numerators and color factors. 
depicted in Fig. 1(c), which we refer to as a double pentagon. Here, we content ourselves with the details that are directly relevant for the computation of the symbol of the $\mathcal{N}=4$ SYM amplitude.

Genuine five-point functions depend on five independent Mandelstam invariants, $s_{12}, s_{23}, s_{34}, s_{45}, s_{51}$, with $s_{i j}=2 p_{i} \cdot p_{j}$. We also find the parity-odd invariant $\epsilon_{5}=$ $\operatorname{tr}\left[\gamma_{5} \not \not \not_{4} \not \not_{5} \not \not_{1} \not \not_{2}\right]$ useful. Its square can be expressed in terms of $s_{i j}$ through $\Delta=\left(\epsilon_{5}\right)^{2}$, with the Gram determinant $\Delta=\left|2 p_{i} \cdot p_{j}\right|$, with $1 \leq i, j \leq 4$.

The integrals of the double-pentagon topology can be related through integration-by-parts relations to a basis of 108 master integrals, which were calculated using the differential equations method [34,35]. In doing this, it was crucial to identify a good basis [35,36], namely, a basis of integrals with uniform transcendental weight (UT integrals): Taking into account a conventional overall normalization [extracting a factor $\exp \left(-\gamma_{\mathrm{E}} \epsilon\right) g^{2} /(4 \pi)^{2-\epsilon}$ per loop], the order- $1 / \epsilon^{4}$ terms of such integrals are constant, the order$1 / \epsilon^{3}$ terms are given by onefold integrals (logarithms), and, in general, the order- $\epsilon^{-4+n}$ terms are given by $n$-fold iterated integrals.

With this choice of basis, the differential equations assume their canonical form [35]:

$$
\vec{d}\left(s_{i j} ; \epsilon\right)=\epsilon\left(\sum_{k=1}^{31} a_{k} d \log W_{k}\left(s_{i j}\right)\right) \vec{I}\left(s_{i j} ; \epsilon\right),
$$

where $a_{k}$ are $108 \times 108$ rational-number matrices, and $W_{k}$ are the so-called symbol letters, algebraic functions of the kinematics encoding the branch-cut structure of the master integrals. The emerging symbol alphabet coincides with the 31-letter alphabet conjectured in Ref. [29], and is obtained by closing under all permutations of the external momenta the 26-letter alphabet relevant for the planar master integrals [25].

The master integrals of this canonical basis are thus given by the so-called pentagon functions, i.e., iterated integrals in the 31-letter alphabet of [29].

The construction of the canonical basis was achieved by combining three cutting-edge strategies. The algorithmic search for $d \log$ integrands, having rational-number leading singularities [28,36], was in fact supplied, for the highest sector, with two novel methods: a $D$-dimensional analysis of Gram determinants and the module lift computation in algebraic geometry. A thorough discussion is contained in [22].

Once the differential equations (1) and the value of $\vec{I}$ at some boundary point are known, the problem of evaluating the master integrals $\vec{I}$ at any kinematic point in a Laurent expansion around $\epsilon=0$ is solved [35]. The boundary values can be determined analytically from physical consistency conditions, as discussed in [28]. In particular, if one is only interested in the symbol [4] of the master integrals $\vec{I}$, the boundary values are needed only at the leading order in the $\epsilon$ expansion, i.e., only at order $1 / \epsilon^{2 \ell}$ for a $\ell$-loop integral. Obtaining the beyond-the-symbol terms requires applying the method of solving the differential equations of $[22,28]$ for all permutations of the integrals appearing in the amplitude, which is beyond the scope of the present paper. As was already observed for the other two top topologies, the symbols of the master integrals of the double pentagon satisfy the second entry condition conjectured in Ref. [29].

Calculation of the amplitude.-The integrand for the full five-point two-loop amplitude in $\mathcal{N}=4$ SYM was constructed in [33] using color-kinematics duality and $D$-dimensional generalized unitarity cuts. In terms of the diagrams shown in Fig. 1, its expression is very compact,

$\mathcal{A}_{5}^{(2)}=\sum_{S_{5}}\left(\frac{I^{(a)}}{2}+\frac{I^{(b)}}{4}+\frac{I^{(c)}}{4}+\frac{I^{(d)}}{2}+\frac{I^{(e)}}{4}+\frac{I^{(f)}}{4}\right)$,

where the sum runs over all permutations of the external legs. This representation of the integrand is valid in $D=$ $4-2 \epsilon$ dimensions, in the regularization scheme where external states and momenta live in $D=4$ dimensions, and the internal momenta are $D$ dimensional.

We reduce the diagrams in Eq. (2) to the basis of UT integrals for the three top topologies shown in the first row of Fig. 1. The basis integrals are then substituted with the corresponding symbols, and the permutations are carried out at the symbol level.

Note that, while having the advantage of being valid in $D$ dimensions, the diagrams, figuring in Eq. (2), do not have uniform transcendental weight. This complexity in the intermediate stages contrasts with an expected simplicity in the final structure: MHV amplitudes are in fact conjectured to have uniform transcendental weight $[7,13,37,38]$, and it is known [39] that their leading singularities [40] are given by Parke-Taylor tree-level super-amplitudes [41,42] only,

$$
\mathrm{PT}\left(i_{1} i_{2} i_{3} i_{4} i_{5}\right)=\frac{\delta^{8}(Q)}{\left\langle i_{1} i_{2}\right\rangle\left\langle i_{2} i_{3}\right\rangle\left\langle i_{3} i_{4}\right\rangle\left\langle i_{4} i_{5}\right\rangle\left\langle i_{5} i_{1}\right\rangle},
$$

where $\delta^{8}(Q)$ is the super-momentum conservation delta function. Reference [43] provides a representation of the four-dimensional integrand where this property is manifest.

Furthermore, the diagrams in (2) are expressed in terms of MHV prefactors called $\gamma_{i j}$ in [33], rather than PT factors. The individual $\gamma_{i j}$, however, cannot be uniquely rewritten in terms of PT factors, thus making such structure even more obscure.

In order to suppress the proliferation of spurious rational functions, and to overcome the difficulty in translating the individual $\gamma_{i j}$ MHV prefactors to PT factors, we exploit the insight we have in the structure of the final function and adopt the following approach. 
While performing the permutations and the sum in Eq. (2), we substitute the kinematic variables with random numbers in the rational prefactors. Then, we single out the prefactor of each individual symbol in the amplitude, and match it with an ansatz made of a $\mathbb{Q}$-linear combination of six independent PT factors. Following [43], we use a basis of the following six Parke-Taylor factors:

$$
\begin{array}{ll}
\mathrm{PT}_{1}=\mathrm{PT}(12345), & \mathrm{PT}_{2}=\mathrm{PT}(12354), \\
\mathrm{PT}_{3}=\mathrm{PT}(12453), & \mathrm{PT}_{4}=\mathrm{PT}(12534), \\
\mathrm{PT}_{5}=\mathrm{PT}(13425), & \mathrm{PT}_{6}=\mathrm{PT}(15423) .
\end{array}
$$

Finally, the coefficients of the ansätze for the rational prefactors of the individual symbols appearing in the amplitude are fixed entirely by considering six random sets of kinematics. Additional sets are used to validate the answer.

After summing over all permutations, therefore, the underlying simplicity of the full amplitude emerges: All spurious rational functions cancel out, and the amplitude turns out to be a linear combination of UT integrals, with prefactors given by PT tree-level super-amplitudes.

The amplitude is a vector in color space. The color structures of the diagrams in Eq. (2) are obtained by associating a structure constant $i \sqrt{2} f^{a b c}$ with each trivalent vertex in Fig. 1. We prefer to expand the amplitude in a basis $\left\{\mathcal{T}_{\lambda}\right\}$ of 12 single traces, $\lambda=1, \ldots, 12$, and 10 double traces, $\lambda=13, \ldots, 22$, defined in Eqs. (2.1) and (2.2) of [44]. For example,

$$
\begin{aligned}
\mathcal{T}_{1} & =\operatorname{Tr}(12345)-\operatorname{Tr}(15432), \\
\mathcal{T}_{13} & =\operatorname{Tr}(12)(\operatorname{Tr}(345)-\operatorname{Tr}(543)),
\end{aligned}
$$

where $\operatorname{Tr}\left(i_{1} i_{2} \ldots i_{n}\right)$ denotes the trace of the generators $T^{a}$ of the fundamental representation of $S U\left(N_{c}\right)$ normalized as $\operatorname{Tr}\left(T^{a} T^{b}\right)=\delta^{a b}$. The other color basis elements $\mathcal{T}_{\lambda}$ are given by permutations of $\mathcal{T}_{1}$ and $\mathcal{T}_{13}$.

Adopting the conventions of Ref. [44], we decompose the amplitude as follows:

$\mathcal{A}_{5}^{(2)}=\sum_{\lambda=1}^{12}\left(N_{c}^{2} A_{\lambda}^{(2,0)}+A_{\lambda}^{(2,2)}\right) \mathcal{T}_{\lambda}+\sum_{\lambda=13}^{22}\left(N_{c} A_{\lambda}^{(2,1)}\right) \mathcal{T}_{\lambda}$.

All partial amplitudes $A_{\lambda}^{(2, k)}$ exhibit the elegant structure discussed above:

$$
A_{\lambda}^{(2, k)}=\frac{1}{\epsilon^{4}} \sum_{w=0}^{4} \epsilon^{w} \sum_{i=1}^{6} \mathrm{PT}_{i} f_{w, i}^{(k, \lambda)}+\mathcal{O}(\epsilon),
$$

where $\mathrm{PT}_{i}$ are the $\mathrm{PT}$ factors defined by Eqs. (4), and $f_{w, i}^{(k, \lambda)}$ are weight- $w$ symbols.

Our result was validated through a series of strong checks, which we describe below.
Color relations: The partial amplitudes $A_{\lambda}^{(2, k)}$ satisfy group-theoretic relations, which automatically follow from rearranging the color structure of the amplitude in the basis $\left\{\mathcal{T}_{\lambda}\right\}$. As a result, the most color-subleading part of the two-loop amplitude $A_{\lambda}^{(2,2)}$ can be rewritten as a linear combination of the planar $A_{\lambda}^{(2,0)}$ and of the double-trace $A_{\lambda}^{(2,1)}$ components [44].

ABDK/BDS ansatz: We verify that the leading-color partial amplitudes $A_{\lambda}^{(2,0)}, \lambda=1, \ldots, 12$, match the formula proposed in Refs. [7,10], and can thus be obtained by exponentiating the one-loop amplitude [45]. The ABDK/ BDS ansatz was previously confirmed numerically [46,47] and was shown to follow from a dual conformal Ward identity [9].

Collinear limit: We consider the limit in which the momenta of two particles, say 4 and 5, become collinear; i.e., we let $p_{4}=z P$ and $p_{5}=(1-z) P$, with $P=p_{4}+p_{5}$. In this limit the two-loop five-point amplitude factorizes into a universal color-blind splitting amplitude and a fourpoint amplitude [48]. Choosing particles 4 and 5 to be positive helicity gluons, we have

$$
\begin{aligned}
\left(\mathcal{A}_{5}^{(2)}\right)^{a_{1}, a_{2}, a_{3}, a_{4}, a_{5}} \stackrel{4 \| 5}{\longrightarrow} & f^{a_{4} a_{5} b}\left[\operatorname{Split}_{-}^{(0)}\left(z ; 4^{+}, 5^{+}\right) \mathcal{A}_{4}^{(2)}\right. \\
& +N_{c} \operatorname{Split}_{-}^{(1)}\left(z ; 4^{+}, 5^{+}\right) \mathcal{A}_{4}^{(1)} \\
& \left.+N_{c}^{2} \operatorname{Split}_{-}^{(2)}\left(z ; 4^{+}, 5^{+}\right) \mathcal{A}_{4}^{(0)}\right]^{a_{1}, a_{2}, a_{3}, b},
\end{aligned}
$$

where $\operatorname{Split}_{-}^{(\ell)}\left(z ; 4^{+}, 5^{+}\right)$and $\mathcal{A}_{4}^{(\ell)}$ are the $\ell$-loop splitting amplitude and four-point amplitude $123 P$, respectively. In order to control the collinear limit $4 \| 5$, we introduce a parameter $\delta$, which approaches 0 in the limit, and $y$, which stays finite, and we use the following momentum twistorinspired parametrization for the Mandelstam invariants:

$$
\begin{aligned}
& s_{12}=\frac{s x \sqrt{y}}{x \sqrt{y}+\delta(1+x)+\delta^{2} \sqrt{y}(1+x)} \\
& s_{23}=s x \\
& s_{34}=\frac{s z}{1+(1+x) \sqrt{y}(1-z) \delta}, \\
& s_{45}=\frac{s x(1+x) \sqrt{y} \delta^{2}}{x \sqrt{y}+\delta(1+x)+\delta^{2} \sqrt{y}(1+x)} \\
& s_{15}=\frac{s x(1-z)}{1+(1+x)(1-z) \sqrt{y} \delta}
\end{aligned}
$$

where $s, t$ are Mandelstam invariants of the four-point amplitude $123 P$, and $x=t / s$. Substituting the parametrization (9) into the letters of the pentagon alphabet, and expanding them up to the leading order in $\delta$, yields a 14letter alphabet. Note, however, that the right-hand side of Eq. (8) contains only the letters $\{\delta, s, x, 1+x, z, 1-z\}$. 
The symbol of the four-point amplitude in fact belongs to the alphabet $\{x, 1+x\}$, and the loop corrections of the splitting factors are specified by the alphabet $\{z, 1-z\}$. This means that the majority of the 14-letter alphabet has to drop out in the collinear limit, thus making this cross-check very constraining. We use the two-loop splitting amplitudes given in [48], and the four-point amplitude up to $\mathcal{O}\left(\epsilon^{2}\right)$ from [21], and find perfect agreement with Eq. (8).

Infrared dipole formula and hard remainder function:

Up to two loops, the IR singularities of gauge-theory scattering amplitudes of massless particles factorize according to the dipole formula [49-52]

$$
\mathcal{A}\left(s_{i j}, \epsilon\right)=\mathbf{Z}\left(s_{i j}, \epsilon\right) \mathcal{A}^{f}\left(s_{i j}, \epsilon\right),
$$

where the factor $\mathbf{Z}\left(s_{i j}, \epsilon\right)$ captures all IR singularities, and $\mathcal{A}^{f}$ is thus a finite hard part of the five-point amplitude $\mathcal{A} \equiv \mathcal{A}_{5}$. We use bold letters to indicate operators in color space. Since we are interested in the symbol of the amplitude, we omit all beyond-the-symbol terms in the following formulas. The factor $\mathbf{Z}\left(s_{i j}, \epsilon\right)$ is then given by

$$
\mathbf{Z}\left(s_{i j}, \epsilon\right)=\exp g^{2}\left(\frac{\mathbf{D}_{0}}{2 \epsilon^{2}}-\frac{\mathbf{D}}{2 \epsilon}\right),
$$

where $\mu$ is a factorization scale, and the dipole operators acting on pairs of incoming particles are defined by

$$
\mathbf{D}_{0}=\sum_{i \neq j} \overrightarrow{\mathbf{T}}_{i} \cdot \overrightarrow{\mathbf{T}}_{j}, \quad \mathbf{D}=\sum_{i \neq j} \overrightarrow{\mathbf{T}}_{i} \cdot \overrightarrow{\mathbf{T}}_{j} \log \left(-\frac{s_{i j}}{\mu^{2}}\right),
$$

with $\mathbf{T}_{i}^{b} \circ T^{a_{i}}=-i f^{b a_{i} c_{i}} T^{c_{i}}$.

Let us denote by $\mathcal{A}_{; w}^{(\ell)}$ the weight- $w$ part of the $\ell$-loop amplitude, which is of order $\epsilon^{w-2 \ell}$ in the $\epsilon$ expansion of $\mathcal{A}^{(\ell)}$. Then, we find that the IR-divergent terms of $\mathcal{A}^{(2)}$ are completely determined by the lower-loop data as dictated by the dipole formula (10),

$$
\begin{aligned}
& \mathcal{A}_{; 0}^{(2)}=\frac{25}{2} N_{c}^{2} \mathcal{A}^{(0)}, \quad \mathcal{A}_{; 1}^{(2)}=\frac{5}{2} N_{c} \mathbf{D} \mathcal{A}^{(0)}, \\
& \mathcal{A}_{; 2}^{(2)}=\frac{1}{8}[\mathbf{D}]^{2} \mathcal{A}^{(0)}+5 N_{c} \mathcal{A}_{; 2}^{(1)}, \\
& \mathcal{A}_{; 3}^{(2)}=\frac{1}{2} \mathbf{D} \mathcal{A}_{; 2}^{(1)}+5 N_{c} \mathcal{A}_{; 3}^{(1)},
\end{aligned}
$$

and the two-loop correction $\mathcal{H}^{(2)}$ to the IR-safe hard function $\mathcal{H}\left(s_{i j}\right) \equiv \lim _{\epsilon \rightarrow 0} A^{f}\left(s_{i j}, \epsilon\right)$ is given by

$$
\mathcal{A}_{; 4}^{(2)}=\mathcal{H}^{(2)}+5 N_{c} \mathcal{A}_{; 4}^{(1)}+\frac{1}{2} \mathbf{D} \mathcal{A}_{; 3}^{(1)} .
$$

We note that the symbol of $\mathcal{H}^{(2)}$ does not depend on $W_{31}$.

The two-loop double-trace part of the hard function $\mathcal{H}\left(s_{i j}\right)$ is the new piece of information. The IR poles and the leading-color components of the amplitude are in fact entirely determined by lower loop information through the dipole formula (10) and the ABDK/BDS ansatz [7,10], respectively. Moreover, the most-subleading-color part can be obtained from the leading-color and the double-trace components via color relations [44]. Only the double-trace part of the hard function can be considered as new, and it is therefore worth looking for a more compact representation of it.

We find the following concise formula,

$$
\mathcal{H}_{\mathrm{dbl}-\mathrm{tr}}^{(2)}=\sum_{S_{5}}\left[N_{c} \mathcal{T}_{13} \mathrm{PT}_{1} g_{\text {seed }}^{(4)}\right]
$$

where $g_{\text {seed }}^{(4)}$ is a weight- 4 symbol, $\mathrm{PT}_{1}$ is defined by Eq. (4), and $\mathcal{T}_{13}$ is defined in Eq. (5). We provide the expression of $g_{\text {seed }}^{(4)}$ split into parity-even and parity-odd parts in the Supplemental Material Hdt_seed_even.txt and Hdt_seed_odd.txt [53], respectively.

Multi-Regge limit.-We now study the multi-Regge limit $[54,55]$ of the amplitude in the physical $s_{12}$ channel,

$$
s_{12} \gg s_{34}>s_{45}>0, \quad s_{23}<s_{15}<0 .
$$

We parametrize the kinematics in this limit as

$$
\begin{aligned}
& s_{12}=s / x^{2}, \quad s_{34}=s_{1} / x, \quad s_{45}=s_{2} / x, \\
& s_{23}=t_{1}, \quad s_{15}=t_{2},
\end{aligned}
$$

and let $x \rightarrow 0$. Substituting this parametrization in the pentagon alphabet, and expanding up to the leading order in $x \rightarrow 0$, we find that it reduces significantly and factorizes into the tensor product of four independent alphabets: $\{x\}$, $\{\kappa\},\left\{s_{1}, s_{2}, s_{1}-s_{2}, s_{1}+s_{2}\right\},\left\{z_{1}, z_{2}, 1-z_{1}, 1-z_{2}, z_{1}-z_{2}\right.$, $\left.1-z_{1}-z_{2}\right\}$, where $\kappa, z_{1}$, and $z_{2}$ are defined as

$\kappa=\frac{s_{1} s_{2}}{s}, \quad t_{1}=-\kappa z_{1} z_{2}, \quad t_{2}=-\kappa\left(1-z_{1}\right)\left(1-z_{2}\right)$.

The two one-letter alphabets simply correspond to powers of logarithms. The third alphabet corresponds to harmonic polylogarithms [56], and the fourth to two-dimensional harmonic polylogarithms [57].

The Regge limit of the single-trace leading-color terms has already been studied [5]. The simple form of the ABDK/BDS formula $[7,10]$ at five points, consisting only of logarithms, is in fact Regge exact.

We are now, for the first time, in the position to take the multi-Regge limit of the double-trace subleading-color part of the hard function $\mathcal{H}_{\mathrm{dbl}-\mathrm{tr}}^{(2)}$, and we find that it vanishes at the symbol level. It will be interesting to investigate whether this remains true at the function level.

We can also go further and consider the subleading power corrections to $\mathcal{H}_{\mathrm{dbl}-\text { tr }}^{(2)}$, of which we present analytically the leading-logarithmic contribution 


$$
\begin{aligned}
\mathcal{H}_{\mathrm{dbl} \text {-tr }}^{(2)} \underset{x \rightarrow 0}{\longrightarrow} \frac{2}{3} x \log ^{4}(x)\left(\frac{\kappa z_{2}}{s_{1}}\left(11\left(\mathcal{T}_{15}+\mathcal{T}_{19}\right)-4 \mathcal{T}_{14}\right)\right. \\
\left.+\frac{\kappa\left(1-z_{1}\right)}{s_{2}}\left(11\left(\mathcal{T}_{16}+\mathcal{T}_{21}\right)-4 \mathcal{T}_{17}\right)\right) .
\end{aligned}
$$

We provide the weight- 4 symbol of the first subleading power corrections to $\mathcal{H}_{\mathrm{dbl}-\text { tr }}^{(2)}$ in the Supplemental Material subleading_multi_Regge.txt [53].

Conclusions and outlook.-In this Letter, we computed, for the first time, the symbol of a two-loop five-particle amplitude analytically. The infrared divergent part of our result constitutes a highly nontrivial check of the two-loop dipole formula for infrared divergences, leading to the first analytic check of two-loop infrared factorization for five particles. Our result provides a substantial amount of analytical data for future studies. For example, we started the analysis of the multi-Regge limit at subleading color. We found that the leading power terms vanish, and we provided the subleading terms. Further terms can be straightforwardly obtained from our symbol. We observed that the nonplanar pentagon alphabet implies a simple structure of the Regge limit. It will be interesting to understand whether this alphabet is also sufficient to describe five-particle scattering at higher loop orders. It will also be relevant to explore whether hints of directional dual conformal symmetry [31,58,59], which is present at the level of individual integrals, can be found at the level of the full amplitude, and whether there is a connection to Wilson loops [60].

We thank V. Mitev for collaboration in early stages of this work. This research received funding from the Swiss National Science Foundation (Ambizione Grant No. PZ00P2161341), the European Research Council (ERC) under the European Union's Horizon 2020 Research and Innovation Programme (Grant Agreement No. 725110), "Novel Structures in Scattering Amplitudes." J. H., Y.Z., and S.Z. also wish to thank the Galileo Galilei Institute for hospitality during the workshop "Amplitudes in the LHC Era."

Note added.-Recently, Ref. [61] appeared, in which the authors use another set of master integrals to calculate the symbol of the two-loop five-point amplitude in $\mathcal{N}=4 \mathrm{SYM}$, in agreement with our result.

[1] J. M. Drummond, J. Henn, G. P. Korchemsky, and E. Sokatchev, Nucl. Phys. B828, 317 (2010).

[2] J. M. Drummond, J. M. Henn, and J. Plefka, J. High Energy Phys. 05 (2009) 046.

[3] N. Berkovits and J. Maldacena, J. High Energy Phys. 09 (2008) 062.

[4] A. B. Goncharov, M. Spradlin, C. Vergu, and A. Volovich, Phys. Rev. Lett. 105, 151605 (2010).
[5] J. Bartels, L. N. Lipatov, and A. Sabio Vera, Phys. Rev. D 80, 045002 (2009).

[6] W. L. van Neerven, Z. Phys. C 30, 595 (1986).

[7] Z. Bern, L. J. Dixon, and V. A. Smirnov, Phys. Rev. D 72, 085001 (2005).

[8] J. M. Drummond, J. Henn, V. A. Smirnov, and E. Sokatchev, J. High Energy Phys. 01 (2007) 064.

[9] J. M. Drummond, J. Henn, G. P. Korchemsky, and E. Sokatchev, Nucl. Phys. B826, 337 (2010).

[10] C. Anastasiou, L. Dixon, Z. Bern, and D. A. Kosower, Phys. Rev. Lett. 91, 251602 (2003).

[11] J. M. Drummond, J. Henn, G. P. Korchemsky, and E. Sokatchev, Nucl. Phys. B815, 142 (2009).

[12] Z. Bern, L. J. Dixon, D. A. Kosower, R. Roiban, M. Spradlin, C. Vergu, and A. Volovich, Phys. Rev. D 78, 045007 (2008).

[13] L. J. Dixon, J. M. Drummond, and J. M. Henn, J. High Energy Phys. 11 (2011) 023.

[14] L. J. Dixon, J. M. Drummond, and J. M. Henn, J. High Energy Phys. 01 (2012) 024.

[15] L. J. Dixon, J. M. Drummond, M. von Hippel, and J. Pennington, J. High Energy Phys. 12 (2013) 049.

[16] L. J. Dixon, M. von Hippel, and A. J. McLeod, J. High Energy Phys. 01 (2016) 053.

[17] L. J. Dixon, J. Drummond, T. Harrington, A. J. McLeod, G. Papathanasiou, and M. Spradlin, J. High Energy Phys. 02 (2017) 137.

[18] J. Drummond, J. Foster, O. Gurdogan, and G. Papathanasiou, arXiv:1812.04640.

[19] S. Caron-Huot, L. J. Dixon, A. McLeod, and M. von Hippel, Phys. Rev. Lett. 117, 241601 (2016).

[20] J. Golden, A. B. Goncharov, M. Spradlin, C. Vergu, and A. Volovich, J. High Energy Phys. 01 (2014) 091.

[21] J. M. Henn and B. Mistlberger, Phys. Rev. Lett. 117, 171601 (2016).

[22] D. Chicherin et al., arXiv:1812.11160.

[23] T. Gehrmann and E. Remiddi, Nucl. Phys. B601, 248 (2001).

[24] T. Gehrmann and E. Remiddi, Nucl. Phys. B601, 287 (2001).

[25] T. Gehrmann, J. M. Henn, and N. A. Lo Presti, Phys. Rev. Lett. 116, 062001 (2016); 116, 189903(E) (2016).

[26] C. G. Papadopoulos, D. Tommasini, and C. Wever, J. High Energy Phys. 04 (2016) 078.

[27] T. Gehrmann, J. M. Henn, and N. A. Lo Presti, J. High Energy Phys. 10 (2018) 103.

[28] D. Chicherin, T. Gehrmann, J. M. Henn, N. A. Lo Presti, V. Mitev, and P. Wasser, J. High Energy Phys. 03 (2019) 042.

[29] D. Chicherin, J. Henn, and V. Mitev, J. High Energy Phys. 05 (2018) 164.

[30] D. Chicherin, J. M. Henn, and E. Sokatchev, Phys. Rev. Lett. 121, 021602 (2018).

[31] D. Chicherin, J. M. Henn, and E. Sokatchev, J. High Energy Phys. 09 (2018) 012.

[32] S. Abreu, B. Page, and M. Zeng, J. High Energy Phys. 01 (2019) 006.

[33] J. J. Carrasco and H. Johansson, Phys. Rev. D 85, 025006 (2012).

[34] T. Gehrmann and E. Remiddi, Nucl. Phys. B580, 485 (2000). 
[35] J. M. Henn, Phys. Rev. Lett. 110, 251601 (2013).

[36] P. Wasser, M.Sc. thesis, 2016, https://publications.ub.unimainz.de/theses/frontdoor.php?source_opus=100001967.

[37] N. Arkani-Hamed et al., Grassmannian Geometry of Scattering Amplitudes (Cambridge University Press, Cambridge, England, 2016).

[38] A. V. Kotikov and L. N. Lipatov, Nucl. Phys. B769, 217 (2007).

[39] N. Arkani-Hamed, J. L. Bourjaily, F. Cachazo, A. Postnikov, and J. Trnka, J. High Energy Phys. 06 (2015) 179.

[40] F. Cachazo, arXiv:0803.1988.

[41] S. J. Parke and T. R. Taylor, Phys. Rev. Lett. 56, 2459 (1986).

[42] V. Nair, Phys. Lett. B 214, 215 (1988).

[43] Z. Bern, E. Herrmann, S. Litsey, J. Stankowicz, and J. Trnka, J. High Energy Phys. 06 (2016) 098.

[44] A. C. Edison and S. G. Naculich, Nucl. Phys. B858, 488 (2012).

[45] Z. Bern, L. J. Dixon, and D. A. Kosower, Phys. Rev. Lett. 70, 2677 (1993).

[46] Z. Bern, M. Czakon, D. A. Kosower, R. Roiban, and V. A. Smirnov, Phys. Rev. Lett. 97, 181601 (2006).

[47] F. Cachazo, M. Spradlin, and A. Volovich, Phys. Rev. D 74, 045020 (2006).

[48] Z. Bern, L. J. Dixon, and D. A. Kosower, J. High Energy Phys. 08 (2004) 012.

[49] S. Catani, Phys. Lett. B 427, 161 (1998).
[50] S. M. Aybat, L. J. Dixon, and G. F. Sterman, Phys. Rev. Lett. 97, 072001 (2006).

[51] S. M. Aybat, L. J. Dixon, and G. F. Sterman, Phys. Rev. D 74, 074004 (2006).

[52] Ø. Almelid, C. Duhr, and E. Gardi, Phys. Rev. Lett. 117, 172002 (2016).

[53] See Supplemental Material at http://link.aps.org/ supplemental/10.1103/PhysRevLett.122.121602, for the seed symbol defined in Eq. (15), and the first subleading power correction to the double-trace two-loop hard function in the multi-Regge limit Eq. (16).

[54] E. A. Kuraev, L. N. Lipatov, and V. S. Fadin, Zh. Eksp. Teor. Fiz. 71, 840 (1976) [Sov. Phys. JETP 44, 443 (1976)].

[55] V. Del Duca, arXiv:hep-ph/9503226.

[56] E. Remiddi and J. A. M. Vermaseren, Int. J. Mod. Phys. A 15, 725 (2000).

[57] T. Gehrmann and E. Remiddi, Comput. Phys. Commun. 144, 200 (2002).

[58] Z. Bern, M. Enciso, H. Ita, and M. Zeng, Phys. Rev. D 96, 096017 (2017).

[59] Z. Bern, M. Enciso, C.-H. Shen, and M. Zeng, Phys. Rev. Lett. 121, 121603 (2018).

[60] R. Ben-Israel, A. G. Tumanov, and A. Sever, J. High Energy Phys. 08 (2018) 122.

[61] S. Abreu, L. J. Dixon, E. Herrmann, B. Page, and M. Zeng, following Letter, Phys. Rev. Lett. 122, 121603 (2019). 